\title{
Similarity assessment between local regions of 3D CAD model based on tree kernel
}

\author{
Haonan Luo ${ }^{a}$, Jing Bai ${ }^{\text {b, * }}$ \\ School of Computer Science and Engineering, Beifang University of Nationalities, Yinchuan 750021, \\ China \\ a582152339@qq.com, bbaijing_nun@163.com
}

Keywords: 3D CAD model, local regions, extended feature tree, convolution tree kernel, similarity assessment.

\begin{abstract}
To effectively characterize the topology and geometric information of 3D CAD models, trees, graphs and other non-linear data structures have become the important descriptors of 3D CAD models. However, the matching based on tree or graph is still the main bottleneck of 3D CAD model similar evaluation. In this paper, a tree kernels based similar evaluation algorithm is proposed. With extended feature trees as the feature descriptors of local regions, the method uses convolution operation to break down the extended feature tree into sub-structure, and the final evaluation of extended feature trees is achieved by the matching between their sub-structures. The method ensures the polynomial matching time, improves the retrieval accuracy by refining the matching size. Experimental results demonstrate the effectiveness of the algorithm.
\end{abstract}

\section{Introduction}

3D CAD model local searching is an important research topic in the field of CAD. Generally speaking, it is necessary to simultaneously portray topology and geometry information of 3D CAD local regions so as to effectively characterize their features, thus it is necessary to extract nonlinear structure descriptors such as tree or graph. However, the matching process of such descriptors is still a complex issue, and for most of time, it is difficult to find the balance between speed and accuracy for the matching. The existing similarity evaluation scheme based on the nonlinear feature descriptors includes hierarchical graph method [1], method based on the random walk [2], the spread of skeleton-based approach [3] and progressive method proposed by Zhang [4].These method using enumeration or approximate skill to insure efficiency or accuracy at the single side, and can't meet the demand of retrieve user.

Convolution tree kernel is a special convolution kernel [5], which is widely used in the field of natural language processing [6]. For 3D model retrieval based on tree feature descriptor, the kernel has a great potential to apply, but based on some known difficulties, there are still no related work. Therefore, this paper proposes a similarity evaluation algorithm for local regions of 3D CAD models based on convolution tree kernel, the algorithm hierarchically completes the matching between large and small structures, which greatly reduces the number of matching programs, so it is possible to ensure the polynomial match time efficiency; meanwhile, the method can ensure matching accuracy of similar evaluation by refining matching granularity.

\subsection{Representation and extraction of 3D CAD model reusable local regions}

The extraction and characterization methods of CAD model reusable local regions are presented in earlier work of my work group [7], here we will not repeat them, but focus on several related concepts:

Design features: it is a features having design semantic.

Extended feature tree: extended feature tree of design feature model is an improved minimum spanning tree of the feature graph $G$ which belongs to this model. Specific: the node of extended feature tree contains all nodes of feature graph; the sides record the parent-child relationship between 
features; Node in the tree contains an attribute values to record the relationship of overlapping or pertinent between current node and others.

Isolated Node: isolated node of extended features tree are such class node or nodes set: they only exist relationships of dependency or independency with other node, but without the relationships of overlapping or pertinent.

Isolated Sub-tree: They are a maximum sub-tree or maximum sub-tree collection rooted at the isolated node except for root node of the extended feature tree.

Furthermore, the extraction of reusable areas and their characterization of extended feature tree is as follow: given a CAD model represented by b-rep, firstly define all design features of model by experts in the field interactively, secondly construct extended feature tree representation of model`s overall structure, then look for all isolated extended sub-tree, and finally extract the reusable local regions and their extended feature tree representations of the model depending on the complexity analysis.

\subsection{Matching and distance calculation based on convolution tree kernel}

The traditional tree matching algorithm requires enumeration of all matching programs, thus the matching efficiency is extremely low; the approximate tree matching algorithms can ensure polynomial matching efficiency, but often greatly reduces the accuracy of similar evaluation. To find a feature tree matching algorithm which let time efficiency and accuracy are both preferred, we introduce the convolution tree kernel distance to the 3D model retrieval domain. Here, convolution kernel uses a similar operation of convolution $\left(^{*}\right)$ to break down larger structures into sub-structure, calculates matching scheme between the cases of the sub-structure, and calculate the similarity of large structures by summing the matching results of sub-structures. Haussler [8] and Watkins [9] have proved that this calculation process meet the symmetry and half positive definitiveness conditions of kernel function; therefore, the similar function constructed in this manner is a kernel function, called the convolution kernel. Specially, the convolution tree kernel is a special convolution kernel existing as tree structure, it calculate the similarity by listing the number of common sub-tree between two trees.

Given two extended feature tree $T_{1}, T_{2}$, the convolution tree kernel distance $K_{\text {СTK }}\left(T_{1}, T_{2}\right)$ between is calculated as follows:

$$
K_{C T K}\left(T_{1}, T_{2}\right)=\sum_{n_{1} \in N_{1}, n_{2} \in N_{2}} \Delta\left(n_{1}, n_{2}\right)
$$

Wherein, $\mathrm{N}_{1}$ and $\mathrm{N}_{2}$ represent the node set included in extended feature tree $\mathrm{T}_{1}, \mathrm{~T}_{2}, \Delta\left(n_{1}, n_{2}\right)$ is the distance of sub-trees which took root in $\mathrm{n}_{1}$ and $\mathrm{n}_{2}$, the calculation of $\Delta\left(n_{1}, n_{2}\right)$ is as follows:

$<1>$ If the topological structure of two extended feature tree which took root in $n_{1}$ and $n_{2}$ are not exactly identical, $\Delta\left(n_{1}, n_{2}\right)=0$, and the calculation is finished; otherwise, go to step $<2>$;

$<2>$ calculating as follows:

$$
\Delta\left(n_{1}, n_{2}\right)=\lambda \prod_{k=1}^{\# \operatorname{ch}\left(n_{1}\right)}\left(1+\Delta\left(\operatorname{ch}\left(n_{1}, k\right), \operatorname{ch}\left(n_{2}, k\right)\right)\right)
$$

Wherein, \# ch (n) represents the number of sub-tree rooted in node $n, c h(n, k)$ is the k-th sub-tree rooted in node $n$. Here we require, $c h(n, k)$ must contain two or more nodes, $\lambda(0<\lambda<1)$ is a recession factor to gain a balance between different sizes of the sub-tree. $\Delta\left(\operatorname{ch}\left(n_{1}, k\right), \operatorname{ch}\left(n_{2}, k\right)\right)$ to characterize distance of the $k$-th sub-tree rooted in node $n_{1}$ between $k$-th sub-tree rooted in node $n_{2}$, from step $<1>$ we can find that these matched sub-tree have totally same topological structure:

Here, distance between sub-tree $\Delta\left(\operatorname{ch}\left(n_{1}, k\right), \operatorname{ch}\left(n_{2}, k\right)\right)$ is defined as the weighted sum of distance of all matched nodes, as follows:

$$
\Delta\left(\operatorname{ch}\left(n_{1}, k\right), \operatorname{ch}\left(n_{2}, k\right)\right)=\sum_{(u, f(u)) \in f} w(u)^{*} D(u, f(u))
$$

Wherein, $w(u)$ is weight of node $u$, which characterize the right of distance of node $u$ and $f(u)$ in whole similar evaluation, $D(u, f(u))$ represent the distance between node $u$ and $f(u)$, which is defined as follows: 


$$
D(u, f(u))=\omega_{r} * D_{b}(u, f(u))+\omega_{n} * D_{t}(u, f(u)) *\left(\alpha D_{v}(u, f(u))+\beta D_{f}(u, f(u))\right)
$$

Wherein, $D_{b}$ is distance of brother relationship between node $u$ and $f(u), D_{t}$ is distance of node type, $D_{v}$ is distance of model volume, $D_{f}$ is distance of face number.

Based on above algorithm and formulas, we can easily calculate the similarity between two given extended feature tree.

\section{Experiment and analysis}

The paper takes Microsoft Visual Studio 2003 as integrated development environment, HOOPS, ACIS as graphics architecture. In the experiment, there are 43 common 3D CAD model as input, by constructing the corresponding design feature model and extract reusable regions, we got a model library contains 117 reusable local regions.

To demonstrate the superior performance of this similarity evaluation algorithm in terms of search accuracy, several existing evaluation algorithms and the evaluation algorithm proposed in this paper were compared in the way of recall-precision curve, the result is presented in Figure1.

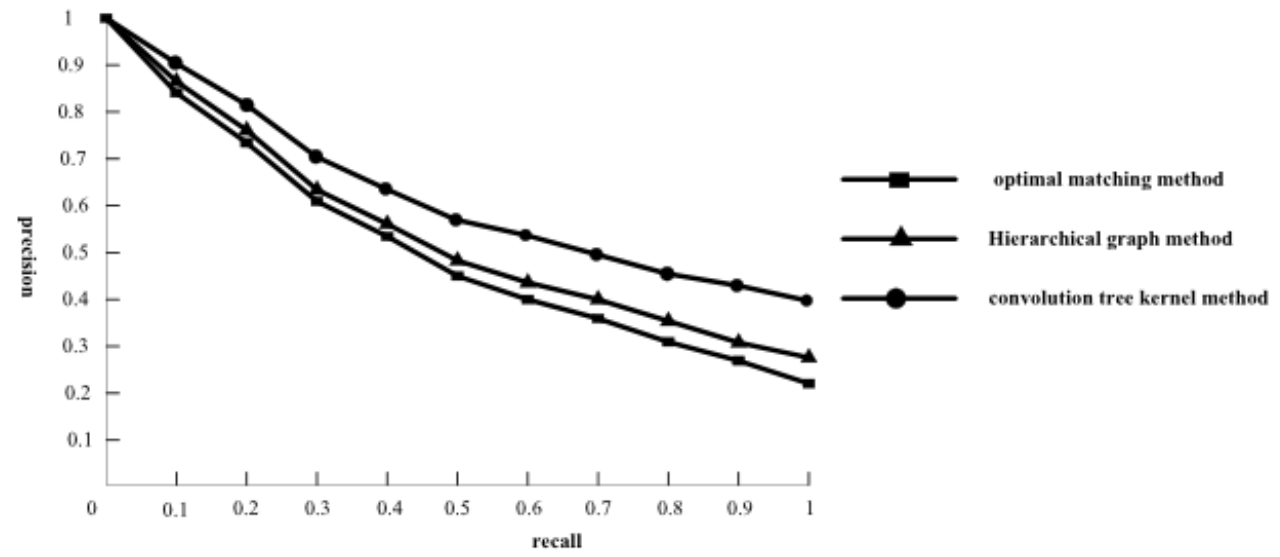

Figure1. Recall-precision curve

The algorithm is also advantageous in terms of time efficiency. We have constructed several model library containing 500, 700, 1000, 1500...10000 reusable local regions and use them as input running program on the PC in the hardware configuration for the Intel Core i7-2600K CPU @ 3.40GHz,3.0G memory. We count the running time when calculating convolution tree kernel distance matrix between every reusable local regions in model library, shown in Figure 2.

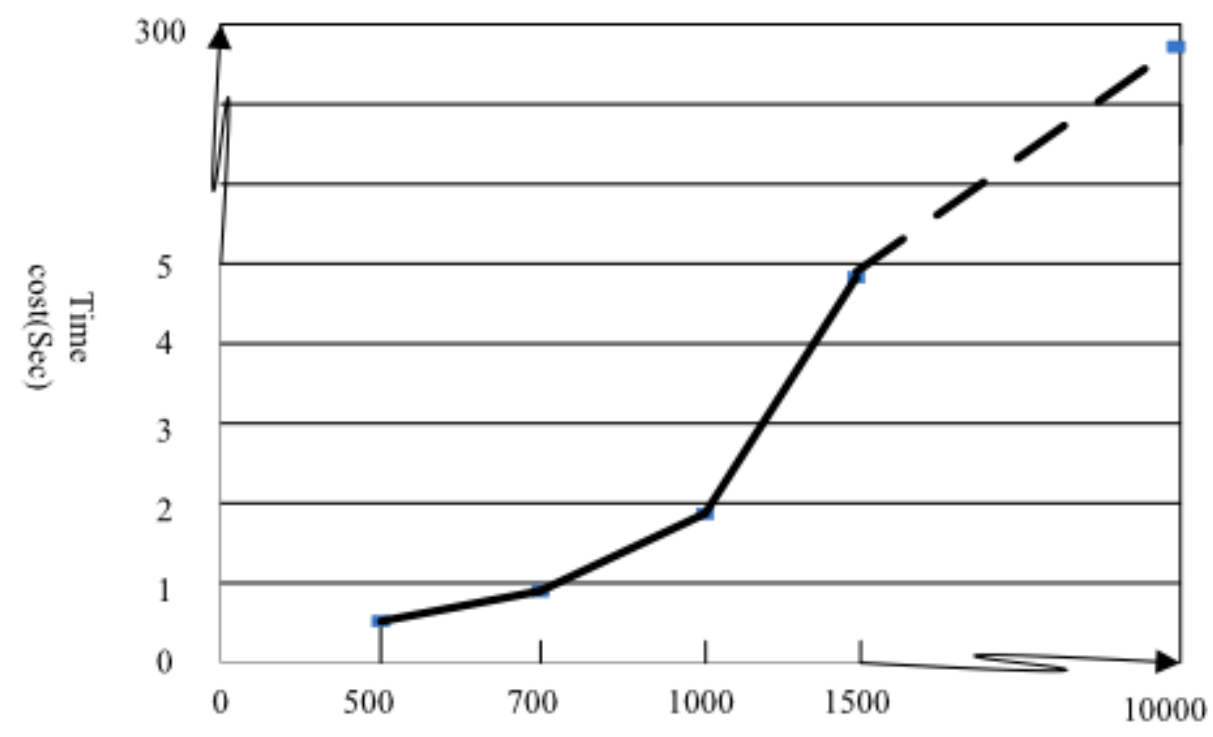

Figure2. Time cost curve 


\section{Summary}

This paper proposes a similarity evaluation algorithm of 3D CAD model local region based on convolution tree kernel, gives the extended feature tree characterization and extraction process of model local regions, and describes how to dismantle the large tree to small structure and complete matching and distance calculation process. The proposed similar evaluating method can significantly improve time efficiency and accuracy of model local regions retrieval, and it has also guiding significance of other entity retrieval using tree feature descriptor; the retrieval test of 3D CAD local regions and related experiments verified the effectiveness of the algorithm.

\section{Acknowledgement}

Supported by the National Natural Science Foundation of China under Grant No. 61163016

\section{References}

[1] Jing Bai, Weihua Tang, Yusheng Liu, Shuming Gao. Hierarchical Graph Generation and Efficient Matching for Solid Model Similarity Assessment. JOURNAL OF COMPUTERAIDED DESIGN \& COMPUTER GRAPHICS. Vol. 21 (2009) No. 7, p. 869-879.

[2] Weihua Tang, Yusheng Liu. Similarity assessment of 3D CAD model based on random walks graph matching. Journal of Mechanical \& Electrical Engineering. Vol. 27 (2010) No. 7, p. 43-46.

[3] Master's thesis:(Wei Gao:3D CAD Model Similarity Estimation Method based on Dilation(master degree, Zhejiang University, China,2006).p.23)

[4] Zhiyong Zhang, Zhigeng Pan, Li Li. Progressive Similarity Matching Algorithm for 3D Models. JOURNAL OF COMPUTER- AIDED DESIGN \& COMPUTER GRAPHICS. Vol. 17 (2005) No. 8, p. $1760-1765$.

[5] Michael Collins, Nigel Duffy. Convolution Kernels for Natural Language. Proc. of the NIPS.2001, p. 625-632.

[6] Fang Kong, Guodong Zhou. Pronoun Resolution in English and Chinese Languages Based on Tree Kernel. Journal of Software. Vol. 23 (2012) No. 5, p. 1085-1099.

[7] Jing Bai.3D CAD Model similarity assessment based on extended feature tree. Computer Integrated Manufacturing Systems. Vol. 20 (2014) No. 2, p. 267-275.

[8] Haussler D. Convolution kernels on discrete structures. Technical Report, UCSCCRL-99-10, Santa Cruz: University of California at Santa Cruz, 1999.

[9] Watkins C. Dynamic alignment kernels. Technical Report, CSD-TR-98-11, Royal Holloway, University of London, 1999. 